\title{
Long-term Carotid Access in the Goat: Observations on Application of a Totally Implantable Catheter System
}

The investigation of metabolism of individual organs and tissues in vivo requires simultaneous measurements of arterio-venous concentration differences. Long-term metabolic studies require frequent and convenient access to veins and arteries of the relevant organs or tissues, and if blood samples are obtained by repeated veni - or arteriopuncture, difficulties may arise due to vessel thrombosis and an increasing apprehension of the goat during time. Arterial punctures are considered more painful than venipuncture, according to human patients. In the conscious goat, arteriopuncture may induce bleating, dilatation of the pupil, increased heart rate, blood glucose and free fatty acid levels, which may bias the results of a metabolic study (Linzell 1963). Furthermore, it is important to avoid situations which contribute negatively to the welfare of the goat. Establishment of permanent exteriorized carotid loops does not eliminate these problems and has a disadvantage of being vulnerable to injury. Jha et al. (1961) reported that 3 out of 10 goats died from haemorrhages, due to loop injury. The carotid artery may surgically be relocated to a subcutaneous position, which makes it less vulnerable, but also less accessible for percutaneous puncture (Tavenor 1969). Our experience with such subcutaneous relocated artery loops is that scar tissue formation is so strong after being catheterized 3-4 times, that further catheterization requires specialized assistance. The artery is eventually closed by fibrous tissue. An alternative to the repeated percutaneous puncture of subcutaneously relocated artery loops is the establishment of chronic indwelling arterial catheters, where the tip of the catheter is exteriorized to the skin. This method is well described (Huntington et al. 1989), but requires a dressing of the exteriorisation site, and restrain of the goat, in order to protect the tip from being pulled out. Therefore, we describe a modification of a totally implantable catheter system, developed for human intra-arterial long-term delivery of drugs, for permanent catheterization of the common carotid artery of goats. The entire access system of this catheter is under the skin.

The catheter set (Port-A-Cath ${ }^{\circledR}$ No. 21-205104, Pharmacia A/S, Denmark) consisted of a soft radioopaque silicone rubber catheter (outer diameter $2.3 \mathrm{~mm}$, inner diameter $0.8 \mathrm{~mm}$, length $760 \mathrm{~mm}$ ), a stainless steel housing (the portal) with a self-sealing silicone septum, accessible by percutaneous needle puncture, and a device to ensure connection of the catheter to the portal (Fig. 1). A suture bead was located 10 $\mathrm{cm}$ from the distal tip, but we found it unsuitable for proper securing of the catheter to the arterial wall and adventitia. Therefore, in advance of surgery, a sterile silicone cuff was made from a $1 / 2 \mathrm{~cm}$ long piece of silicone tubing (Silastic $^{\mathrm{R}}$, inner diameter $2.5 \mathrm{~mm}$, outer diameter $3.2 \mathrm{~mm}$ ). A purse string suture with a non- 


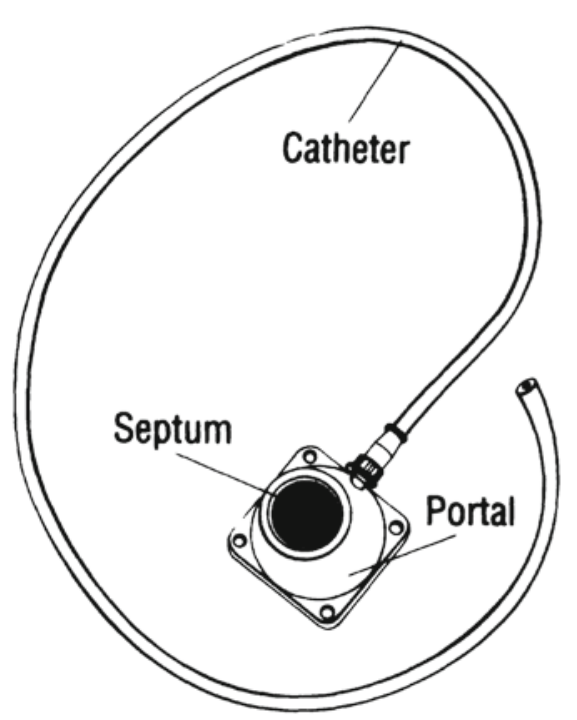

Figure 1. Schematic drawing of the implantable catheter system. The portal is located subcutaneously.

resorbent suture was preplaced close to the end of the cuff, leaving the needle on the suture. The cuff was finally glued to the site of the suture bead with the purse string suture towards to the tip of the catheter.

Surgery was carried out under general anaesthesia. A skin incision was made immediately dorsal to the raised jugular vein. The incision was approximately $8 \mathrm{~cm}$ long and located on the middle of the jugular furrow. The sternocephalicus muscle was separated bluntly from the omohyoideus muscle and the carotid artery exposed. The artery was separated from the carotid sheath and the introducer needle was inserted downstream into the artery. A teflon coated guidewire, length $120 \mathrm{~cm}$, diameter 0.71 $\mathrm{mm}$, with a $3 \mathrm{~cm}$ soft tip was advanced approximately $10 \mathrm{~cm}$. A vessel screw dilator (9 French, $25 \mathrm{~cm}$ ) was then introduced a few centimeters in order to expand the puncture site.
After removal of the vessel dilator, the catheter was threaded over the guide wire until the cuff laid firmly against the arterial adventitia. Haemorrhage was controlled by gentle compression. The patency of the catheter was checked by aspiration of blood and the catheter was then filled with heparinized saline (100 units $/ \mathrm{ml})$ and plugged with the cap from the catheter set. The cuff was anchored into the tissue using the free ends of the preplaced purse string suture. The free end of the catheter was then pulled through a small piece of a monofilament net (2 $\times 2 \mathrm{~cm}$ ), which did not allow passage of the cuff. The net was then sutured to the surrounding tissue. This was done as an extra precaution to avoid pulling through of the catheter. A second skin incision was made at the preferred site of insertion of the portal and subcutaneous tunneling to the dorsal part of the neck was done with a suture needle, model Bühner. Care should be taken not to kink or twist the catheter. We strongly recommend not to place extra sutures e.g. to keep the catheter in a smooth curve. A subcutaneous pocket was then made at the second incision, the portal was filled with saline as described by the manufacturer and attached to an appropriate length of the catheter, leaving adequate slack to enable body movement (approximately $5 \mathrm{~cm}$ extra catheter). The portal should be placed at least $1.5 \mathrm{~cm}$ from the skin incision. Patency should be checked before closing of both skin incisions.

After return of the swallowing reflex, pethidine was administered. Administration was repeated every 6th hour during the first $24 \mathrm{~h}$ and later if the animal showed signs of pain. Treatment with a non-steroidal anti-inflammatory drug was indicated, but not initiated, because the goats were intended for studies involving measurement of prostanoids. Penicillin was administered pre-operatively and for 6 days after the surgery. All goats were relatively unaffected by the operation and ate within a few hours after 
withdrawal of anaesthesia. Sutures were removed after 10 days.

The goats were kept in loose housing, without restrain or collars.

The catheters were flushed according to the recommandations of the manufacturer on day 1,2, 4 and 6 after surgery. Later, flushing was performed once a week with saline containing 100 units of heparin.

We have implanted 19 arterial catheters. We did not observe complications such as purulent inflammation, implant rejection, sepsis or erosions of the implant through the skin, which are common when catheters are exteriorized through skin. However, some swelling and accumulation of serous fluid occurred in a few cases around the portals during the first days. In 2 cases, the fluid was aspirated aseptically with a $20 \mathrm{G}$ needle and a syringe, whereafter the implant healed.

The most common complication was pulling out of the catheter at the site of entry into the artery. This happened several times before we mounted a stronger cuff and the monofilament net. These events usually happened after about a week and was not associated with fatal bleeding, in most cases not even with subcutaneous haematomas.

Catheters, implanted as described, generally were patent for several months. A mean function time could not be established, since the goats were euthanized for other reasons, when the catheters were still patent. The reason of failure of 4 catheters was fibrin sheath forma- tion at the catheter tip. In 2 cases, the artery occluded during time. The goats were not clinically affected by this process. Inadvertent catheter withdrawal owing to snagging, a common cause of catheter failure, was not observed in this study, because of the subcutaneous position of the catheter. Arterial blood could be sampled without apprehension of the goat. In conclusion, care should be taken when the catheter is sutured to the arterial wall. Advantages of the totally implantable system were improved animal welfare due to reduction of stress during the blood sampling procedure, combined with a minimal risk of infection and fatal haemorrhage.

P. Haubro Andersen ${ }^{1}$, M.O. Nielsen ${ }^{2}$ and J. Fjeldborgl

${ }^{1}$ Departments of Clinical Studies and ${ }^{2}$ Anatomy \& Physiology, Royal Veterinary and Agricultural University, Frederiksberg, Denmark.

\section{References}

Huntington, GB, Christopher KR, Stroud, BH: Techniques for Measuring Blood Flow in Splanchnic Tissues of Cattle. J. Dairy Sci. 1989, 72, 15831595.

Jha SK, Lumb WV, Johnston RF: Establishment of permanent carotid loops in goats. Amer. J. vet. Res. 1961, 22, 948-949.

Linzell JL: Carotid loops. Am. J. vet. Res. 1963, 24, 223-224.

Tavenor WD: Technique for the subcutaneous relocation of the common carotid artery in the horse. Amer. J. vet. Res. 1969, 30, 1881-1883.

(Received January 6, 1995):

Reprints may be obtained from: P. Haubro Andersen, Department of Clinical Studies, Surgery, The Royal Veterinary and Agricultural University, Bülowsvej 13, DK-1870 Frederiksberg C, Denmark. 
